\title{
Cdk5 in Neuroskeletal Dynamics
}

\author{
Deanna Smith \\ Department of Biological Sciences, University of South Carolina, Columbia, S.C., USA
}

\section{Key Words}

Cdk5 - Tau - MAP2 - Nudel - Amyloid precursor protein .

Presenilin-1 • $\beta$-Catenin · Pak1 - CAMKII · Cables •

Disabled $\cdot$ Amphiphysin $1 \cdot$ Synapsin 1

\begin{abstract}
Signal transduction in a broad sense refers to intracellular, molecular changes triggered by cues outside the cell in order to bring about behavioral responses such as migration, differentiation, secretion, or death. Until recently, the best-studied end targets of signal transduction pathways were transcriptional regulators that contribute to changes in gene expression. However, it is clear that many pathways can act through mechanisms other than, or in addition to, transcription control. The three major cytoskeletal systems, microtubules, intermediate filaments, and filamentous actin, are targets for this kind of regulation. Acute changes in cytoskeletal organization are particularly important during embryonic development, when many cells are motile and undergo dramatic changes in shape. Cytoskeletal dynamics are also important in motile cells in adult systems and, in a more limited way, in cells that carry out rapid, regulated secretion. Even changes in synaptic efficacy may involve structural modifications requiring changes in the neuroskeleton. Cytoskeletal reorganization can itself be viewed as signal transduction, producing changes in
\end{abstract}

molecular trafficking and interactions. This review considers evidence that cyclin-dependent kinase 5 is a modulator of neuroskeletal dynamics.

Copyright $(2003$ S. Karger AG, Basel

\section{Introduction}

The term 'cytoskeleton' evokes a rigid structure that maintains the cell's shape, much like the bones of an animal. It stems from early microscopic studies in which the filaments in fixed samples appeared static. In the last 3 decades, this purely structural view of the cytoskeleton has been displaced by evidence that cytoskeletal systems are highly dynamic and can undergo rapid reorganization globally or locally, depending on the age, the environment, or the function of a particular cell. In neuronal development, for example, establishment of polarity, migration, neurite outgrowth, and synaptogenesis despend on cytoskeletal dynamics. Some of the dynamic processes that have been documented are listed in figure 1. Modifications of these processes probably function in mature systems and may influence synaptic function and neuronal survival. Large numbers of proteins are being identified that have roles in regulating cytoskeletal dynamics during mitosis and migratory behavior [1-3]. Many of these are targeted by cyclin-dependent kinase (Cdk) family members [4], including Cdk5. The Cdk5 activity de-

\section{KARGER \\ Fax +41613061234 \\ E-Mail karger@karger.ch \\ www. karger.com

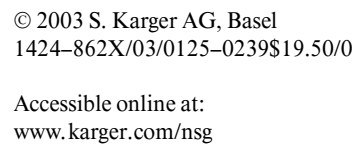

Deanna Smith

Department of Biological Sciences, University of South Carolina

607 Coker Life Sciences Building, 700 Sumter Street

Columbia, SC 29208 (USA)

Tel. +1 803777 3020, Fax +1 803777 4002, E-Mail deannasm@biol.sc.edu 
Fig. 1. The importance of neuroskeletal dynamics. Various degrees of cytoskeletal reorganization are accomplished through a group of related but distinct mechanisms (top box). The regulation of these events is critical for virtually all aspects of neuronal development and function (bottom box). The molecular machinery regulating neuroskeletal dynamics must be tightly regulated, and $\mathrm{CDk} 5$ stands poised to be a part of the orchestration.

Fig. 2. Cdk5 is intimately linked to receptor systems and substrates that can impact neuroskeletal dynamics. In pink are proteins that can act as Cdk5 substrates. In green are proteins that are linked to cdk5 regulation or that may be influenced by Cdk5 activity by virtue of their interaction with Cdk5 substrates. Proteins are placed in categories according to which neuroskeletal network they are most notably associated with, although substantial cross talk occurs across categories.
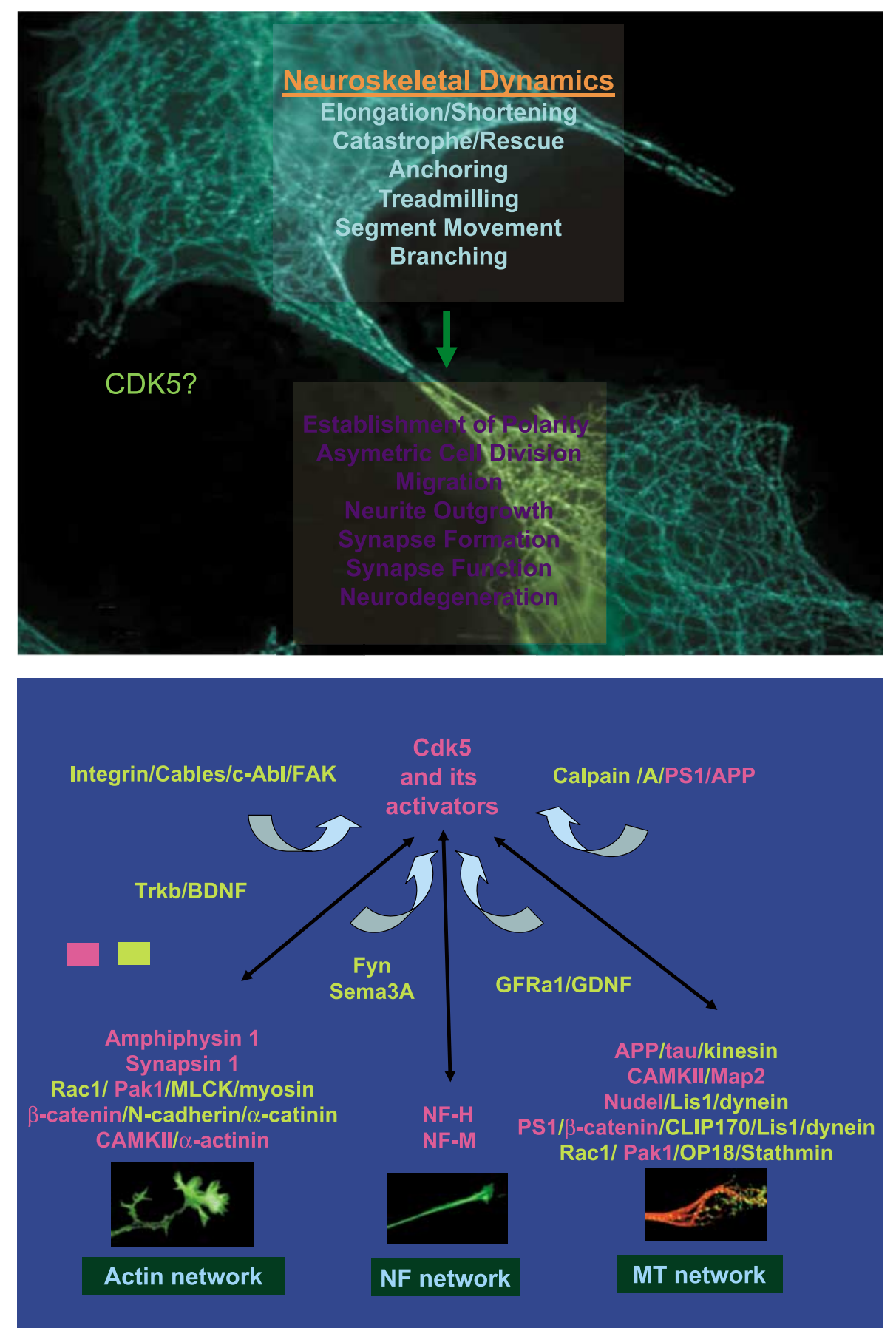

pends not on cyclins, like the activity of other Cdk family members, but on two largely neuronal proteins, p35 and p39 [5]. Because of this, the Cdk5 activity is most prominent in developing neurons, where it functions in neuronal migration and neuritic growth and synaptogenesis. Some of the known and suspected neuroskeletally related
Cdk5 substrates are collected in figure 2. In adult animals, Cdk5 may modulate synaptic transmission [6]. Although Cdk5 is vital during development, its activity at the wrong place or at the wrong time in mature systems is implicated in neurodegenerative disorders like Alzheimer's disease [7] and amyotrophic lateral sclerosis (ALS) [8]. 
A number of cell surface receptors involved in adhesion, pathfinding, and neurotransmission have been linked with Cdk5 activity [5]. Some, including integrin receptors, plexins, neurotrophin receptors, and neurotransmitter receptors, regulate the kinase. Others respond to Cdk5 activity. Cell surface receptors often interact directly or indirectly with cytoskeletal elements, and because many Cdk5 substrates are associated with cytoskeletal dynamics, it is worth considering a role for Cdk5 in the communication between receptors and neuroskeletal systems. Among known or suspected Cdk5 substrates are microtubule-associated proteins (MAPs), including tau [9], MAP2 [Tsai, personal commun.], and the dyneinassociated protein, Nudel [10]. Cdk5 substrates also include a long list of proteins related to actin dynamics, such as CaMKII [11], the Abl-interacting protein, Cables [12], Pak1 [13], amphiphysin [14], and synapsin 1 [15]. Finally, Cdk5 can use two of three neuronal intermediate filament subunits, NF-H and NF-M, as substrates. It is not too farfetched, therefore, to speculate that a component of Cdk5's signaling function may be to coordinate motile behaviors through its impact on, or sensitivity to, cytoskeletal dynamics.

This review will concentrate on $\mathrm{Cdk} 5$ data related to each of the three cytoskeletal networks, considering them separately with the understanding that a large degree of molecular cross talk occurs between the different networks.

\section{The Microtubule Connection}

The microtubule network can be stabilized or broken down rapidly (catastrophe). The rate of polymerization of $\alpha-$ and $\beta$-tubulin dimers can also be regulated. Microtubule stability is supported by several MAPs [2]. The wellknown MAPs, MAP2 and tau, bind to the surface of the microtubule, bridging tubulin subunits, and may neutralize the negative charge on the microtubule surface. Members of the XMAP215/Stu2p/TOG family promote microtubule polymerization at 'plus' ends and regulate the catastrophe frequency [16]. The microtubule-destabilizing factor katanin severs microtubules [17], while depolymerizing kinesins of the KinI family may cause protofilament peeling [18]. Op18/Stathmin may sequester tubulin dimers or promote GTP hydrolysis to destabilize microtubules [19]. Lis 1 interacts with the microtubule motor, cytoplasmic dynein, and can reduce microtubule catastrophe frequency [20] and impact microtubule organization [21]. Cytoplasmic dynein also promotes transport of assembled microtubule segments. This function is particularly prominent in axons [22]. There is evidence that dynein can also directly influence the polymerization dynamics of microtubules. At the vertebrate kinetochore, for example, motors interact directly with microtubule ends and modulate polymerization dynamics to orchestrate chromosome movements during mitosis [23]. The Lis1-associated protein Nudel may impact dynein behavior and microtubule organization [10, 24]. End-binding proteins emphasize and make use of the intrinsic polarity of tubulin polymers $[25,26]$. CLIP-170 and EB1 copolymerize with new tubulin subunits by binding to a special configuration of the microtubule plus end. They also seem to serve as links between growing microtubules and kinetochores or focal adhesions centers at the cell cortex through proteins such as APC and $\beta$-catenin [27, 28]. Moreover, EB1 interacts with cytoplasmic dynein, and binding of $\beta$-catenin to the cytoplasmic dynein/dynactin motor complex at microtubule ends and cortical sites or kinetochores can stabilize microtubules [28]. Many of the factors regulating the microtubule stability are themselves regulated by kinases and phosphatases. New findings point to a molecular interrelationships between different skeletal systems. In this first section, we will concentrate on some of these microtubule-regulatory proteins and their relationship to Cdk5.

\section{Tau}

In 1993, Baumann et al. [9] described a link between Cdk5 and microtubules [9]. Cdk5 copurified with known MAPs and the kinase was capable of phosphorylating tau, a fairly well-studied and somewhat infamous MAP. Abnormally hyperphosphorylated forms of tau were known to form paired helical filaments typical of neurofibrillary tangles in the brains of Alzheimer's disease patients [9]. Over the last decade, a strong link has been forged between Cdk5 deregulation and Alzheimer's disease [7]. Neurotoxic induction of calpain leads to the proteolytic cleavage of p35 to $\mathrm{p} 25$ [29]. This product maintains its ability to activate Cdk5, but is more stable and less membrane associated [30]. Interestingly, Alzheimer's-like tau phosphorylation is more apparent when Cdk5 is paired with p25. Cotransfection of Cdk5 and p25 resulted not only in a dramatic increase in tau phosphorylation and in the disorganization of microtubules, but was more toxic than the coexpression of Cdk5 and p35 [31]. The brains of mice in which a p25 transgene was expressed showed increased Cdk5 activity, increased immunostaining for hyperphosphorylated tau, axonal swelling, and disorganization of the cytoskeleton [32]. 
Based on these data, most models predict that $\mathrm{Cdk} 5$ phosphorylates tau only under pathological conditions when p25 is generated. However, healthy neurons may have use for tau phosphorylation by $\mathrm{Cdk} 5$ in a spatially or temporally limited manner. So what does tau do in healthy neurons? Tau was originally characterized as a MAP that promoted the assembly of $6 \mathrm{~S}$ tubulin dimers into 36S tubulin rings [33]. Tau was then shown to suppress microtubule catastrophe in vitro [34] and in cultured fibroblasts [35]. Overexpression of tau in $\mathrm{CHO}$ cells had dramatic effects on cellular systems that were thought to be dependent on the function of the microtubule-based motor protein kinesin [36]. These effects were related to the finding that tau overexpression affected the frequency of motor attachment and detachment to microtubule tracks and had an impact on vesicle minus to plus end reversal with a net effect of increasing the directional bias towards minus ends [37]. Tau overexpression also inhibited axon transport in neurons [38].

Despite the obvious impact of tau on microtubules and motors in vitro and in cultured cells, the nervous system of tau-/- mice appears to be normal, and axonal elongation is unaffected in cultured neurons [39]. In some smallcaliber axons, however, the microtubule stability is decreased, and the microtubule organization is significantly altered. An observed increase in expression of another MAP, MAP1A, in these mice suggested that this protein might compensate for the functions of tau in the unaffected large-caliber axons.

The evidence for a nonpathological role for Cdk5 in tau biology is not extensive. However, tau phosphorylation at known Cdk5 sites is decreased in Cdk5-/- mouse brains. This is not the case in p35-/- mouse brains, suggesting that p39 might play a role [40]. Cdk5 is anchored to microtubules through binding to unphosphorylated tau; phosphorylation of tau results in the dissociation of Cdk5. The phosphorylation of tau by $\mathrm{Cdk} 5 / \mathrm{p} 39$ in vitro also decreased tau's affinity for microtubules [40]. Thus, although it has been difficult to pin a physiological function on tau, Cdk5 could conceivably affect normal neuronal cytoskeletal dynamics and motor protein function by regulated posttranslational modification of the protein.

\section{$M A P 2$}

Brain MAP2 was one of the first MAPs to be isolated [41-43]. Whereas tau is preferentially localized to axonal compartments, MAP2 tends to be dendritic. Both proteins are thought to confer stability to microtubules in a phosphorylation-sensitive manner. Like tau, MAP2 can be phosphorylated in vitro by a number of protein kinases, including protein kinases A and C (PKA, PKC) [44], calcium calmodulin dependent kinases (CAMKs) [45], MARK kinases [46], Cdc2 [44], MAP/ERK kinases [47-49], and glycogen synthase kinase $3 \beta$ [50]. Cdk5 can also phosphorylate MAP2 [Tsai, personal commun.]. In general, MAP2 phosphorylation seems to interfere with microtubule association and to decrease the microtubule stability, but the specific effects of individual kinases in particular cellular contexts are not completely understood.

Postsynaptic MAP2 is a target of signaling pathways activated by glutamate [51]. Glutamatergic regulation of MAP2 phosphorylation by CAMKII and PKC may transduce neural activity into modifications in dendritic structure. Indeed, there is a reduction in microtubule density in dendrites and a reduction of dendritic length in MAP2deficient mice [52]. Freda Miller and colleagues [49] recently showed that neuronal activity promoted reversible dendrite formation accompanied by an increased association of MAP2 with microtubules and an increased microtubule stability. Inhibition of either CaMKII or the MEK/ERK pathways was required for dendrite formation, while inhibition of both pathways caused dendrites to retract.

Does Cdk5 play a role in these glutaminergic responses? Cdk5 is enriched in postsynaptic membranes. Moreover, calcium increase or activation of glutamate receptors stimulates an interaction between CAMKII $\alpha$ and Cdk5-regulatory subunits [11]. Inhibition of CAMKII activation diminishes this interaction. Together, these data suggest the intriguing possibility that cross talk between the Cdk5 and CAMKII signal transduction pathways may be a component of the complex molecular/ cytoskeletal mechanisms contributing to synaptic plasticity. It remains to be determined whether Cdk5 is involved in MAP2 regulation in dendrites.

\section{Amyloid Precursor Protein (APP)}

Another link between Alzheimer's disease and Cdk5 was forged by the demonstration that $A \beta$, the proteolytic peptide derived from APP, can induce calpain-dependent cleavage of $\mathrm{p} 35$ and stimulate tau hyperphosphorylation and neurotoxicity [7]. Interestingly, blocking microtubule dynamics with taxol inhibits this $A \beta$-dependent pathway and stabilizes the normally short-lived p35 regulatory subunit [53]. Taxol is not sufficient to protect primary cortical neurons from constitutive Cdk5 activation following overexpression of $\mathrm{p} 25$, however. A nonpathological connection between APP/A $\beta$ and Cdk5 has not been 
described, but there is evidence for APP's involvement in microtubule-based transport. Recent data suggest that it functions as a kinesin-I cargo receptor, mediating the targeting of several synaptic proteins to the nerve terminal [54-57]. Axonal transport of APP is mediated by direct binding to the light-chain subunit of kinesin-I [58]. Moreover kinesin-mediated axonal transport of a membrane compartment containing $\beta$-secretase and presenilin- 1 (PS1) requires APP [59]. Indeed, profound loss of immunoreactivities for the presynaptic proteins synaptophysin and synapsin 1, as well as the dendritic marker MAP2, was reported in many brain areas of APP-/- mice [60]. APP, PS1, synapsin 1, and MAP2 are all likely substrates for Cdk5. Could there be a role for the kinase in microtubule motor-based trafficking of synaptic proteins?

\section{Nudel}

A link between cytoplasmic dynein and tubulin dynamics has been demonstrated in the filamentous fungus Aspergillus nidulans [61]. Temperature-sensitive loss-offunction dynein mutants (NUDA) in Aspergillus showed a lower frequency of microtubule catastrophe, a lower rate of shrinkage during catastrophe, and a lower frequency of rescue. These results indicate that cytoplasmic dynein affects microtubule dynamics in vivo. Loss-of-function mutation in a dynein-interacting protein (NUDF) had similar effects. Haploinsufficiency of the gene encoding the human homolog of NUDF (resulting in a $50 \%$ reduction in protein expression) causes the majority of the known cases of a rare, but severe, developmental brain abnormality termed classical lissencephaly [61]. In animal models of lissencephaly (Lis1+/- mice or flies with Lis1 disrupted in neurons), the neuronal defects are multifold, ranging from mitotic abnormalities in precursors to defects in migration and neuritic growth $[62,63]$. Purified brain Lis 1 has been shown to cosediment with microtubules and to reduce microtubule catastrophe events in vitro [20]. Furthermore, changes in the expression level of Lis 1 in cultured nonneuronal cells has a dramatic effect on microtubule organization [21]. Dynein and Lis1 are linked to Cdk5 through a protein called Nudel [10, 24] and probably also through a related protein, mNudE [64]. Nudel can be phosphorylated by $\mathrm{Cdk} 5$ in vivo and in vitro. Moreover, phosphospecific antibodies do not recognize Nudel in Cdk5-/- brain extracts [Tsai, personal commun.]. It is not yet clear if and how Lis1 and Nudel regulate dynein function, but it is not out of the question that they regulate dynein's impact on the microtubule stability. Thus Cdk5 might work through Nudel to impact microtubule dynamics.
Lis 1 also seems to be involved in some mitotic roles of dynein [65]. Very recently, phosphorylation of Nudel and mNudE by Cdc2, a mitotic kinase and a close homolog of $\mathrm{Cdk} 5$, was implicated in the translocation of dynein and spindle assembly checkpoint proteins away from kinetochores [66]. The kinase may be regulating a checkpointinactivating event that allows chromosome segregation to occur only when spindles are properly attached. It would be interesting if there were some kind of a parallel checkpoint system that functions to regulate microtubule dynamics during migration.

\section{PS1 and $\beta$-Catenin}

PS1 [see 67 for a recent review] is a component of an enzymatic activity, $\gamma$-secretase, implicated in proteolysis of APP and generation of a form of $A \beta$ peptide abundant in senile plaques. PS1 is produced as a holoprotein, then processed to amino- and carboxy-terminal fragments that are incorporated into stable, high molecular mass complexes. Presenilins function both in Notch signaling (acting as a protease) and in regulating $\beta$-catenin stability in response to Wnt signaling.

Three fairly recent findings are more to the point of this review. PS1 reportedly is a substrate of Cdk5 [68]. Cdk5/p35 phosphorylates a threonine within the carboxyl-terminal domain of PS1 both in vitro and in vivo and stabilizes the carboxy-terminal fragment. Second, PS1 binds to the microtubule plus-end-capping protein, CLIP170 [69]. PS1/CLIP170 interactions have a regulatory effect on $\beta$-amyloid secretion and uptake, but the mechanism is not clear. The authors suggest a scenario in which PS1 guides microtubule binding to specific membrane subdomains possibly containing amyloidogenic fragments. An interesting twist is that CLIP-170 interacts with the Nudel-binding partner, Lis1, in mitotic cells; Lis 1 is apparently responsible for CLIP-170 accumulation at kinetochores [70, 71]. It is not known whether Lis 1 and CLIP-170 interact in neurons. PS1 also interacts with $\beta$ catenin [72], another Cdk5 substrate [73]. Cdk5 apparently phosphorylates $\beta$-catenin to regulate binding of $\beta$-catenin to PS1, but also regulates $\beta$-catenin's interaction with $\mathrm{N}$-cadherin cell adhesion molecules. The likely influence of Cdk5 is downregulation of $\mathrm{N}$-cadherin at the cell surface $[74,75]$. Interestingly, an intracellular pool of $\mathrm{N}$-cadherin is tightly associated with the microtubule network [151]. Moreover, expression of N-cadherins in centrosome-free cytoplasts increased levels of microtubule polymer and also changed the behavior of microtubules from treadmilling to dynamic instability [152]. N-cadherins thus appear to initiate a signaling pathway that alters 
microtubule organization by stabilizing microtubule ends. Cdk5 may directly or indirectly impact these interactions through regulating $\beta$-catenin's interactions and changing $\mathrm{N}$-cadherin function. Interestingly, Lis 1 and $\beta$ catenin both interact with cytoplasmic dynein and may serve to stabilize microtubules at cortical sites $[28,76$, 77]. A potential role for Cdk5 in a neuronal version of 'microtubule capture' at leading edge or growth cone plasma membranes is intriguing.

Schuman and Murase [75] recently demonstrated that $\mathrm{Cdk} 5$ may regulate a $\beta$-catenin/ $\mathrm{N}$-cadherin interaction in synapses as well. In hippocampal neurons, depolarization caused a decrease in Cdk5 kinase activity and a redistribution of EGFP- $\beta$-catenin from dendritic shaft into spines, where cadherins are highly concentrated. Decreasing the Cdk5 kinase activity with the inhibitor roscovitine caused a similar redistribution of EGFP- $\beta$-catenin into spines and increased the amount of $\beta$-catenin coimmunoprecipitating with cadherin. A point mutation in tyrosine 654 of $\beta$-catenin blocked its movement into spines in response to $\mathrm{Cdk} 5$ inhibition and prevented it from interacting with cadherin.

Although a connection with microtubules in dendritic spines is not likely, Cdk5 is also intimately involved in actin regulation, and $\beta$-catenin interacts with actin through $\alpha$-catenin.

\section{The Actin Connection}

Although Cdk5 in brain extracts cosediments with microtubules after a series of polymerization-depolymerization steps, the Cdk5 immunoreactivity coincides best with regions rich in filamentous actin, such as leading edges of growth cones [78]. Moreover, the Cdk5 activity in nonneuronal cells stimulates changes in actin organization. One of Cdk5's activating partners, p39, colocalizes with actin in cultured cells and coimmunoprecipitates with actin in brain lysates [79]. Disruption of the actin cytoskeleton alters the subcellular distribution of p39 [79].

Like microtubules, actin filament formation and stability is regulated by an extensive collection of actin-binding proteins (over 150) and signal transduction pathways [80]. Some actin-binding proteins maintain the population of assembly-ready actin monomers (profilin). Others bind to and block the growing ends of actin filaments (gelsolin). Actin-binding proteins can nucleate actin assembly (gelsolin, Arp2/3, cofilin), sever actin filaments (gelsolin, ADF/cofilin), or cross-link actin filaments (Arp2/3). The activity of these proteins is regulated by several signaling pathways, most prominently those involving the RHO GTPases. The members of this family of biological switches impact many aspects of actin dynamics and are important in a wide range of cell behaviors [81]. The identification of several Cdk5-interacting proteins/substrates related to actin prompts speculation about a role for $\mathrm{Cdk} 5$ activity in regulating actin dynamics or actin-microtubule interactions. We will consider each of the actin-related Cdk5 links in turn to try to shed some light on how these may relate to the function of the kinase.

\section{Pakl}

The first link between Cdk5 and actin was the demonstration that the kinase phosphorylates Pak1 in a Rac1dependent manner and inhibits Pak1 activity [13]. Rac1 is strongly implicated in actin dynamics and neurite growth, and one of its primary effectors is Pak1 [82]. Strangely, few actin-related targets of Pak1 have been identified, although a recent report [83] suggested that Pak1 could phosphorylate actin directly, resulting in the dissolution of stress fibers and redistribution of microfilaments. Pak1 also targets myosin light-chain kinase and decreases its activity which may have an impact on actin dynamics in migrating cells and growth cones [84]. Finally, Pak1 increases the activity of LIM kinase towards the actin-regulatory protein, cofilin [85].

Although Rac signaling is mainly associated with actin, there is growing evidence that Racl influences actin in response to microtubule dynamics [86]. For example, the growth of microtubules induced in fibroblasts by removal of the microtubule destabilizer nocodazole activates Rac1 GTPase, leading to the polymerization of actin in lamellipodial protrusions [87]. It was also recently shown that the Pak activity might inhibit a microtubule-destabilizing protein, Op18/Stathmin [88], so Rac/Pak signaling could influence microtubule dynamics as well. Pak1 phosphorylated on T212 (the Cdk5 phosphorylation site) is enriched in axonal growth cones and colocalizes with small peripheral bundles of microtubules [89]. Cortical neurons overexpressing a Pak1 mutated at T212 display a tangled neurite morphology which suggests that the microtubule cytoskeleton is affected. In the developing cortex and in cultured fibroblasts, phosphorylated Pak1 T212 is enriched in microtubule-organizing centers and along parts of mitotic spindles. Indeed, cyclin $\mathrm{B} 1 / \mathrm{Cdc} 2$ phosphorylates Pak1 during mitosis. Banjeree et al. [90] showed that, in living cells, a peptide mimicking phosphorylated T212 accumulates at the centrosomes and spindles and causes an increased length of astral microtubules during meta- 
phase or following nocodazole washout. Staining of fibroblasts extracted with detergent using anti-Pak1 antibodies revealed that a small subpopulation of this kinase remained tightly bound to the mitotic spindles. Exposure to a Pak1 peptide phosphorylated by Cdk5, but not unphosphorylated peptide, reduced the pool of microtubulebound Pak1. Significant levels of the phosphorylated peptide were tightly bound to the cytoskeleton, suggesting that it competes with endogenous Pak1 for microtubule association.

Thus, accumulating data suggest that reciprocal Cdk5 and Pak1 activity could be important in regulating actin/ microtubules, but the specifics of this cooperation are not yet well understood.

\section{$\alpha$-Actinin and CaMKII}

CaMKII is localized in the postsynaptic density (PSD) and is necessary for LTP induction. The autophosphorylated state of CaMKII binds tightly to NMDA receptors and to $\alpha$-actinin [91]. $\alpha$-Actinins are primarily thought of as actin cross-linking proteins that tether cell surface receptors, ion channels, and adhesion complexes to the cortical actin cytoskeleton [92-97]. $\alpha$-Actinin is present in PSDs and dendritic spines [98]. Disruption of the actin cytoskeleton attenuates NMDA-receptor-mediated transmission [99] and causes the loss of CaMKII at the PSD and $\alpha$-actinin at spines [100].

$\mathrm{Cdk} 5 / \mathrm{p} 35$ and Cdk5/p39 can complex with both CaMKII and $\alpha$-actinin-1 in a calcium-sensitive manner [11]. Glutamate can strengthen the interaction, to a large extent through NMDA receptors. The association of CaMKII and Cdk5 with $\alpha$-actinin- 1 may serve to anchor both kinases to the subsynaptic cytoskeleton and perhaps modulate their proximity to the NMDA receptor and other substrates at the PSD. Alternatively, neurotransmission may trigger changes in these interactions in order to regulate the underlying actin skeleton. Further studies will be needed to make this distinction. Interestingly, changes in expression or activity of the myosin light-chain kinase, a downstream target of Pak1 kinase, influence NMDA receptor neurotransmission, probably by indirectly regulating actin and myosin [101]. Because the Pak1 activity can be regulated by $\mathrm{Cdk} 5$, it could impact this pathway.

\section{Cables}

Cables is a substrate of the c-Abl tyrosine kinase that promotes the interaction between c-Abl and Cdk5 [12]. Active c-Abl kinase leads to Cdk5 tyrosine phosphorylation, and this phosphorylation is enhanced by Cables. Phosphorylation of Cdk 5 by c-Abl occurs on tyrosine 15
(Y15) which is stimulatory for the $\mathrm{p} 35 / \mathrm{Cdk} 5$ kinase activity. Expression of antisense Cables in primary cortical neurons inhibits neurite outgrowth. Furthermore, expression of active $\mathrm{Abl}$ resulted in lengthening of neurites.

How does this relate to actin dynamics? Actin organization can impact c-Abl activity [102]. c-Abl tyrosine kinase is inhibited by F-actin, and this inhibition can be relieved through mutation of its F-actin-binding domain. In a reciprocal manner, $\mathrm{C}-\mathrm{Abl}$ can impact the actin skeleton. c-Abl stimulates the formation of actin microspikes in fibroblasts spreading on fibronectin and F-actin branches in neurites of rat embryonic cortical neurons [103]. These functions of c-Abl are dependent on its kinase activity, but not on its F-actin-binding domain. It is interesting to speculate that these actions of $\mathrm{c}-\mathrm{Abl}$ might work through its ability to regulate Cdk5.

\section{Disabled 1}

Enabled (Ena) and disabled (Dab) are genetic modulators of the phenotype observed in flies null for the $\mathrm{Abl}$ tyrosine kinase gene [104]. Cdk5 phosphorylates the murine homolog of the Drosophila disabled protein, mDab1, on serine 491 in vitro and in vivo [105]. Mutations in mouse equivalent of disabled have brain abnormalities similar but not identical to Cdk5-/- mice [106, 107]. Although the functional significance of $\mathrm{mDab} 1$ phosphorylation by Cdk5 is not clear, there are interesting links between mDab1 and another Cdk5 substrate, APP. The cytosolic domain of APP interacts with mDab1 and another protein, FE56 [108, 109]. Fe65 functions in the regulation of actin cytoskeleton and cell movement [110]. This may be related to the direct interaction of Fe56 ww domains with the proline-rich repeats in the mouse homo$\log$ of the Drosophila enabled protein (Mena) [111]. Mena is a member of a family of actin-binding proteins that promote or inhibit actin-dependent processes by antagonizing various capping proteins at the barbed end of actin filaments [112]. APP and FE65 colocalize with actin and Mena in lamellipodia of migrating MDCK cells and with $\beta 1$-integrin in focal complexes [110]. Overexpression of APP accelerates cell migration in an MDCK cell woundhealing assay [110]. Coexpression of APP and FE65 dramatically enhances the effect of APP on cell movement, probably by regulating the amount of APP at the cell surface.

In a related role, Fe65 may serve as a linker between another actin-binding protein, c-Abl, and APP. Fe65 binds to the active form of Abl, and APP is tyrosine phosphorylated on Tyr682 in cells expressing this form of $\mathrm{Abl}$ [113]. Active Abl and tyrosine-phosphorylated APP are 
present in coimmunoprecipitated complexes. Together, these findings suggest that Abl, Mena, and mDab1 are involved in APP biology. It will be interesting to determine if and how Cdk5 activity impacts these interactions in neurons.

\section{Amphiphysin 1}

Amphiphysin 1 is generally associated with clathrinmediated endocytosis, but recently has been linked with the actin skeleton, albeit somewhat tenuously [114]. Amphiphysin 1 colocalizes with actin and p35 in neuronal growth cones and is phosphorylated by Cdk5/p35 [14]. Amphiphysin 1 oligonucleotides caused growth cone collapse and disruption of the actin cytoskeleton in cultured hippocampal neurons without affecting receptor-mediated endocytosis. This suggests that amphiphysins could have actin-related functions in growth cones independent of clathrin-mediated endocytosis. In support of this, amphiphysins interact with neuronal isoforms of focal adhesion kinase (FAK) [115]. FAK is a nonreceptor tyrosine kinase that has been shown to be a major mediator of integrin signal transduction pathways. FAK and amphiphysins are associated in nerve terminals, where FAK also interacts with components of the actin cytoskeleton such as talin, $\alpha$-actinin (another Cdk5 substrate), and paxillin and is part of the complex that anchors the Factin cytoskeleton to the plasma membrane [116]. The tangled web gets more tangled when we consider that both FAK and Cdk5 kinase activities can be stimulated by integrin signaling. Is it possible that both FAK and Cdk5 impact cytoskeletal dynamics through amphyphsin 1 and $\alpha$-actinin in response to extracellular matrix interactions? Or does Cdk5 function upstream or downstream of FAK?

\section{Synapsin 1}

Cdk5 reportedly can also phosphorylate synapsin 1 [15], a member of a family of five neuron-specific phosphoproteins associated with the membranes of synaptic vesicles [117]. Synapsins are implicated in controlling neurotransmitter release by tethering vesicles to actin filaments in a phosphorylation-dependent manner, thus regulating the number of vesicles available for release. In living hippocampal terminals, green fluorescent protein labeled synapsin 1a dissociates from synaptic vesicles and disperses into axons during action potential firing, then reclusters to synapses after the cessation of synaptic activity [118]. Synapsin 1 also participates in the formation and maintenance of synaptic contacts in hippocampal neurons. Retarded outgrowth of predendritic neurites and axons from cultured hippocampal neurons was reported in Synapsin 1-/- mice [119]. Furthermore, the synapse formation was significantly delayed, and this impairment was found to be caused by postsynaptic changes [120].

Synapsin 1 can bind to actin [121-128], microtubules [129-132], and neurofilaments [133] in vitro. When bound either to synaptic vesicles or to pure phospholipid vesicles, synapsin 1 exhibits phosphorylation-dependent actin-nucleating activity. Synaptic vesicles depleted of endogenous synapsin 1 decrease rate and extent of actin polymerization. Synapsin 1 is also reportedly an actinbundling protein; this activity is also controlled by phosphorylation [124], but it may be unrelated to the Cdk5 activity [15]. Therefore, the consequence of Cdk5 activity towards synapsin 1 is not clear and will require further study. If indeed synapsin 1 is intimately related to all three neuroskeletal elements, then the possibility exists that it functions in some sort of integrative capacity that could be modulated by Cdk 5 .

\section{The Neurofilament Connection}

Intermediate filament (IF) proteins form the largest family of cytoskeletal proteins in mammalian cells [3]. IF family members include nuclear lamins, vimentin, desmin, glial fibrillary acidic protein, and type I and II keratins. The function of these medium-sized polymers has generally been considered to be structural, but evidence that IFs can modulate essential cell processes and signal transduction events has broadened this view [3].

In neurons, the most prominent IFs are composed of three 'neurofilament' proteins [for two recent reviews see 134, 135]. These are termed NF-H, NF-M, and NF-L to signify high $(\mathrm{H})$, medium $(\mathrm{M})$, and low $(\mathrm{L})$ molecular weight subunits. Neurofilaments possess side arms composed of the $\mathrm{COOH}$ domains of NF-M and NF-H; that of NF-H is heavily phosphorylated in axons. Axonal NF-H becomes highly phosphorylated in maturing systems and is hyperphosphorylated in some neurodegenerative diseases. Neurofilaments have a well-established role in the control of axon caliber. There is also growing evidence based on transgenic mouse studies that neurofilaments can affect the dynamics of microtubules and actin filaments. Neurofilaments contain phosphorylation sites for a large number of protein kinases, including PKA, PKC, ERK, GSK-3 $\beta$, and the stress-activated protein kinase gamma [136]. Cdk5 also figures prominently as a neurofilament kinase [135]. Cdk5 target phosphorylation sites, 
located in tail regions of NF-M and NF-H, consist of the repeat sequence motif, Lys-Ser-Pro (KSP).

Degenerative disorders of motor neurons include a range of progressive fatal diseases such as ALS. Although the causative genetic alterations are known for some cases, the molecular basis of most ALS cases is unknown. However, ALS is characterized by depositions of neurofilaments in the perikarya and proximal axons, and variant alleles of the neurofilament heavy-subunit gene (NF-H) have been found in some ALS patients [136]. Moreover, transgenic mice that overexpress neurofilament proteins show motor neuron degeneration and defects in the axonal transport of neurofilament and other proteins, including tubulin and actin [137]. Mutations in copper/zinc superoxide dismutase 1 (SOD1) can cause ALS through an unidentified mechanism. Two lines of transgenic mice with SOD1 mutations linked to familial ALS showed a reduced transport of selective cargoes, especially tubulin, that arose months before neurodegeneration. For one mutant, this transport defect represents the earliest detectable abnormality [138].

Neurofilament protein is transported in axons as a polymer [139]. Movements are fast and bidirectional, but interspersed with long pauses. Kinesin and cytoplasmic dynein are involved in transporting neurofilaments in axons. Several recent findings have linked motor neuron disease with microtubule motors. Inhibition of dyneinmediated axonal transport by postnatal transgenic overexpression of a component of the dynactin regulatory complex in mouse motor neurons causes neurodegeneration [140]. Interestingly, a missense mutation in another subunit of dynactin has been identified in a human kindred displaying spinal-bulbar muscular atrophy [141]. Missense point mutations in mice that exclusively perturb neuron-specific functions of cytoplasmic dynein result in progressive motor neuron degeneration and Lewy-like inclusion bodies [142]. Finally, mutations in the anterograde kinesin motor, KIF1B, can lead to Charcot-MarieTooth disease, a slowly progressive motor neuropathy [143].

A recent analysis of neurofilament movement in transfected living neurons [144] demonstrated that phosphorylation of NF-H side arms by $\mathrm{Cdk} 5 / \mathrm{p} 35$ influenced the transport of neurofilaments through axons. Phosphorylation effectively slowed neurofilament transport due to increased pausing of neurofilaments. The Cdk5/25 activity is associated with the hyperphosphorylation of tau and neurofilament proteins in mice expressing a mutant SOD1. It has been suggested that perikaryal accumulations of neurofilament proteins in motor neurons may actually lessen the ALS pathogenesis by acting as a phosphorylation sink for this aberrant Cdk5 activity, reducing detrimental hyperphosphorylation of tau and other substrates [145]. However the link between $\mathrm{Cdk} 5$ and neurofilament transport suggests that deregulated $\mathrm{Cdk} 5$ might be the cause of accumulations of neurofilaments. The verdict, then, is still out on a normal physiological role for Cdk5 in neurofilament dynamics.

\section{Concluding Remarks}

The aim of this review is to focus attention on the relationships between $\mathrm{Cdk} 5$ and the neuroskeleton. An emerging theme in cytoskeletal dynamics in all cells is the interrelated nature of the three distinct cytoskeletal elements. For example, we generally think of cell motility as membrane protrusion powered by turnover of adhesion complexes that link the actin cytoskeleton to the extracellular matrix [146]. Directional polarity however, is likely attained by microtubules that modulate Rho GTPase activity and actin behavior at adhesion sites. This sitespecific targeting by microtubules may bring enzymatic complexes (bound to microtubules or MAPs) in close proximity to adhesion complexes to promote complex disassembly and remodeling of the actin cytoskeleton [86].

A requirement for this type of communication would not be unique to neurons, and if we want to make the argument that $\mathrm{Cdk} 5$ serves in an integrative capacity to coordinate neuroskeletal dynamics, we need to explain how these events are coordinated in nonneuronal cells which, for the most part, lack Cdk5 activity. The obvious candidate for a $\mathrm{Cdk} 5$ correlate is the 'mitotic' kinase, $\mathrm{Cdc} 2$, which shares a large degree of homology and substrate specificity with $\mathrm{Cdk} 5$. So, does $\mathrm{Cdc} 2$ participate in cytoskeletal dynamics?

There are actually many parallels. For example, the integrin-mediated $\mathrm{Cdc} 2$ activity has been shown to be important for the migratory activity in prostate cancer cells [147]. Ectopic expression of cyclin A, an activator of $\mathrm{Cdc}$, can confer anchorage-independent mitosis, i.e., uncouple adhesion and cytoskeletal dynamics [148]. Cdc2 can actually exert an effect directly on actin dynamics, through a protein called caldesmon, which inhibits binding of Arp2/3 to F-actin, reducing Arp2/3-mediated nucleation [149]. Phosphorylation of caldesmon with Cdc2 inhibits its ability to interact with actin and reverses its inhibitory effect on Arp2/3-induced actin polymerization. It is not known whether a similar role is played by $\mathrm{Cdk} 5$ in 
neurons. Cdc2 affects MAP2-induced microtubule polymerization in vitro [44] and can phosphorylate Nudel and Pak1 in mitotic cells to regulate dynein and microtubules, respectively $[66,90]$. Cdc2 is also an important regulator of the breakdown of the nuclear membrane in prometaphase. It exerts an impact at the level of nuclear IFs called lamins [150].

We are just beginning to unravel the complexities of coordinated cellular responses to extracellular signals. Our continued effort over the next several years is likely to reveal the extent to which these two Cdk family mem- bers influence cytoskeletal dynamics, and will hopefully shed some light on when and how they do so. Is it a coordinated sweep of enzymatic activity that targets a multitude of substrates in response to specific signals? If so, does it switch rapidly on and off, and do the target substrates fluctuate in response to changing extracellular cues? Alternatively, are cytoskeletal dynamics producing the signals that these kinases are responding to? Answers to these questions will no doubt require the sophisticated proteomic and imaging techniques that are currently being developed and improved at a rapid pace.

\section{References}

1 Chen H, Bernstein BW, Bamburg JR: Regulating actin-filament dynamics in vivo. Trends Biochem Sci 2000;25:19-23.

2 Heald R, Nogales E: Microtubule dynamics. J Cell Sci 2002;115:3-4.

3 Paramio JM, Jorcano JL: Beyond structure: Do intermediate filaments modulate cell signalling? Bioessays 2002;24:836-844.

4 Smith DS, Tsai LH: Cdk5 behind the wheel: A role in trafficking and transport? Trends Cell Biol 2002; 12:28-36.

5 Dhavan R, Tsai LH: A decade of CDK5. Nat Rev Mol Cell Biol 2001:2:749-759.

6 Smith DS, Greer PL, Tsai LH: Cdk5 on the brain. Cell Growth Differ 2001;12:277-283.

7 Lee MS, Tsai LH: Cdk5: One of the links between senile plaques and neurofibrillary tangles? J Alzheimers Dis 2003;5:127-137.

8 Patzke H, Tsai LH: Cdk5 sinks into ALS. Trends Neurosci 2002;25:8-10.

9 Baumann K, Mandelkow EM, Biernat J, et al: Abnormal Alzheimer-like phosphorylation of tau-protein by cyclin-dependent kinases Cdk2 and Cdk5. FEBS Lett 1993;336:417-424.

10 Niethammer M, Smith DS, Ayala R, et al NUDEL is a novel Cdk5 substrate that associates with LIS1 and cytoplasmic dynein. Neuron 2000;28:697-711.

11 Dhavan R, Greer PL, Morabito MA, et al: The cyclin-dependent kinase 5 activators $\mathrm{p} 35$ and p39 interact with the alpha-subunit of $\mathrm{Ca} 2+/$ calmodulin-dependent protein kinase II and alpha-actinin-1 in a calcium-dependent manner. J Neurosci 2002;22:7879-7891.

12 Zukerberg LR, Patrick GN, Nikolic M, et al: Cables links Cdk5 and c-Abl and facilitates Cdk5 tyrosine phosphorylation, kinase upregulation, and neurite outgrowth. Neuron 2000; 26:633-646.

13 Nikolic M, Chou MM, Lu W, et al: The p35/ Cdk5 kinase is a neuron-specific Rac effector that inhibits Pak1 activity. Nature 1998;395: 194-198.
14 Floyd SR, Porro EB, Slepnev VI, et al: Amphiphysin 1 binds the cyclin-dependent kinase (Cdk) 5 regulatory subunit p35 and is phosphorylated by Cdk5 and Cdc2. J Biol Chem 2001;276:8104-8110.

15 Matsubara M, Kusubata M, Ishiguro K, et al: Site-specific phosphorylation of synapsin I by mitogen-activated protein kinase and Cdk5 and its effects on physiological functions. J Biol Chem 1996;271:21108-21113.

16 Shirasu-Hiza M, Coughlin P, Mitchison T: Identification of XMAP2 15 as a microtubuledestabilizing factor in Xenopus egg extract by biochemical purification. J Cell Biol 2003;161: 349-358.

17 McNally KP, Buster D, McNally FJ: Kataninmediated microtubule severing can be regulated by multiple mechanisms. Cell Motil Cytoskeleton 2002;53:337-349.

18 Niederstrasser H, Salehi-Had H, Gan EC, et al: $\mathrm{XKCM} 1$ acts on a single protofilament and requires the $\mathrm{C}$ terminus of tubulin. $\mathrm{J}$ Mol Biol 2002;316:817-828.

19 Andersen SS: Spindle assembly and the art of regulating microtubule dynamics by MAPs and Stathmin/Op18. Trends Cell Biol 2000;10: 261-267.

20 Sapir T, Elbaum M, Reiner O: Reduction of microtubule catastrophe events by LIS1, platelet-activating factor acetylhydrolase subunit. EMBO J 1997;16:6977-6984.

21 Smith DS, Niethammer M, Ayala R, et al: Regulation of cytoplasmic dynein behaviour and microtubule organization by mammalian Lis1. Nat Cell Biol 2000;2:767-775.

22 Baas PW: The role of motor proteins in establishing the microtubule arrays of axons and dendrites. J Chem Neuroanat 1998;14:175180.

23 Hunter AW, Wordeman L: How motor proteins influence microtubule polymerization dynamics. J Cell Sci 2000;113 Pt 24:4379-4389.

24 Sasaki S, Shionoya A, Ishida M, et al: A LIS1/ NUDEL/cytoplasmic dynein heavy chain complex in the developing and adult nervous system. Neuron 2000;28:681-696.
25 Carvalho P, Tirnauer JS, Pellman D: Surfing on microtubule ends. Trends Cell Biol 2003;13: 229-237.

26 Howard J, Hyman AA: Dynamics and mechanics of the microtubule plus end. Nature 2003; 422:753-758.

27 Berrueta L, Tirnauer JS, Schuyler SC, et al: The APC-associated protein EB1 associates with components of the dynactin complex and cytoplasmic dynein intermediate chain. Curr Biol 1999;9:425-428.

28 Ligon LA, Karki S, Tokito M, et al: Dynein binds to beta-catenin and may tether microtubules at adherens junctions. Nat Cell Biol 2001; 3:913-917.

29 Lee MS, Kwon YT, Li M, Peng J, et al: Neurotoxicity induces cleavage of p35 to p25 by calpain. Nature 2000;405:360-364.

30 Patrick GN, Zhou P, Kwon YT, et al: p35, the neuronal-specific activator of cyclin-dependent kinase 5 (Cdk5), is degraded by the ubiquitinproteasome pathway. J Biol Chem 1998;273: 24057-24064.

31 Patrick GN, Zukerberg L, Nikolic M, et al: Conversion of p35 to p25 deregulates Cdk5 activity and promotes neurodegeneration. $\mathrm{Na}$ ture 1999;402:615-622.

32 Ahlijanian MK, Barrezueta NX, Williams RD, et al: Hyperphosphorylated tau and neurofilament and cytoskeletal disruptions in mice overexpressing human p25, an activator of cdk5. Proc Natl Acad Sci USA 2000;97:2910-2915.

33 Weingarten MD, Lockwood AH, Hwo SY, et al: A protein factor essential for microtubule assembly. Proc Natl Acad Sci USA 1975;72: 1858-1862.

34 Drechsel DN, Hyman AA, Cobb MH, et al: Modulation of the dynamic instability of tubulin assembly by the microtubule-associated protein tau. Mol Biol Cell 1992;3:1141-1154.

35 Drubin D, Kobayashi S, Kirschner M: Association of tau protein with microtubules in living cells. Ann N Y Acad Sci 1986;466:257-268. 
36 Ebneth A, Godemann R, Stamer K, et al: Overexpression of tau protein inhibits kinesin-dependent trafficking of vesicles, mitochondria, and endoplasmic reticulum: Implications for Alzheimer's disease. J Cell Biol 1998;143:777_ 794.

37 Trinczek B, Ebneth A, Mandelkow EM, et al Tau regulates the attachment/detachment but not the speed of motors in microtubule-dependent transport of single vesicles and organelles. J Cell Sci 1999; 112:2355-2367.

38 Stamer K, Vogel R, Thies E, et al: Tau blocks traffic of organelles, neurofilaments, and APP vesicles in neurons and enhances oxidative stress. J Cell Biol 2002;156:1051-1063.

39 Harada A, Oguchi K, Okabe S, et al: Altered microtubule organization in small-calibre axons of mice lacking tau protein. Nature 1994; 369:488-491.

40 Takahashi S, Saito T, Hisanaga S, et al: Tau phosphorylation by cyclin-dependent kinase 5/ p39 during brain development reduces its affinity for microtubules. J Biol Chem 2003;278: 10506-10515.

41 Sloboda RD, Dentler WL, Rosenbaum JL: Microtubule-associated proteins and the stimulation of tubulin assembly in vitro. Biochemistry 1976;15:4497-4505.

42 Herzog W, Weber K: Fractionation of brain microtubule-associated proteins: Isolation of two different proteins which stimulate tubulin polymerization in vitro. Eur J Biochem 1978; 92:1-8.

43 Garner CC, Farmer L, Matus A: Single-shot cloning of multiple cDNAs coding for a set of related microtubule-associated proteins. Gene 1988;71:483-490.

44 Itoh TJ, Hisanaga S, Hosoi T, et al: Phosphorylation states of microtubule-associated protein 2 (MAP2) determine the regulatory role of MAP2 in microtubule dynamics. Biochemistry 1997;36:12574-12582.

45 Schulman H: Phosphorylation of microtubuleassociated proteins by a $\mathrm{Ca}^{2+} /$ calmodulin-dependent protein kinase. J Cell Biol 1984;99: 11-19.

46 Ebneth A, Drewes G, Mandelkow E: Phosphorylation of MAP2c and MAP4 by MARK kinases leads to the destabilization of microtubules in cells. Cell Motil Cytoskeleton 1999;44: 209-224.

47 Landreth GE, Smith DS, McCabe C, et al: Characterization of a nerve growth factor-stimulated protein kinase in PC1 2 cells which phosphorylates microtubule-associated protein 2 and pp250. J Neurochem 1990;55:514-523.

48 Hoshi M, Ohta K, Gotoh Y, et al: Mitogen-activated-protein-kinase-catalyzed phosphorylation of microtubule-associated proteins, microtubule-associated protein 2 and microtubuleassociated protein 4 , induces an alteration in their function. Eur J Biochem 1992;203:4352

49 Vaillant AR, Zanassi P, Walsh GS, et al: Signaling mechanisms underlying reversible, activitydependent dendrite formation. Neuron 2002; 34:985-998.
50 Sanchez C, Perez M, Avila J: GSK3beta-mediated phosphorylation of the microtubule-associated protein 2C (MAP2C) prevents microtubule bundling. Eur J Cell Biol 2000;79:252260

51 Quinlan EM, Halpain S: Postsynaptic mechanisms for bidirectional control of MAP2 phosphorylation by glutamate receptors. Neuron 1996;16:357-368.

52 Harada A, Teng J, Takei Y, et al: MAP2 is required for dendrite elongation, PKA anchoring in dendrites, and proper PKA signal transduction. J Cell Biol 2002;158:541-549.

53 Li G, Faibushevich A, Turunen BJ, et al: Stabilization of the cyclin-dependent kinase 5 activator, $\mathrm{p} 35$, by paclitaxel decreases beta-amyloid toxicity in cortical neurons. J Neurochem 2003;84:347-362.

54 Muller U, Kins S: APP on the move. Trends Mol Med 2002;8:152-155.

55 Inomata H, Nakamura Y, Hayakawa A, et al: A scaffold protein JIP-1b enhances amyloid precursor protein phosphorylation by JNK and its association with kinesin light chain 1. J Biol Chem 2003;278:22946-22955.

56 Torroja L, Chu H, Kotovsky I, et al: Neuronal overexpression of APPL, the Drosophila homologue of the amyloid precursor protein (APP), disrupts axonal transport. Curr Biol 1999;9:489-492.

57 Gunawardena S, Goldstein LS: Disruption of axonal transport and neuronal viability by amyloid precursor protein mutations in Drosophila. Neuron 2001;32:389-401.

58 Kamal A, Stokin GB, Yang Z, et al: Axonal transport of amyloid precursor protein is mediated by direct binding to the kinesin light chain subunit of kinesin-I. Neuron 2000;28: 449-459.

59 Kamal A, Almenar-Queralt A, LeBlanc JF, et al: Kinesin-mediated axonal transport of a membrane compartment containing beta-secretase and presenilin-1 requires APP. Nature 2001;414:643-648.

60 Dawson GR, Seabrook GR, Zheng H, et al: Age-related cognitive deficits, impaired longterm potentiation and reduction in synaptic marker density in mice lacking the beta-amyloid precursor protein. Neuroscience 1999;90: $1-13$.

61 Han G, Liu B, Zhang J, et al: The Aspergillus cytoplasmic dynein heavy chain and NUDF localize to microtubule ends and affect microtubule dynamics. Curr Biol 2001;11:719-724.

62 Hirotsune S, Fleck MW, Gambello MJ, et al: Graded reduction of Pafahlb1 (Lis1) activity results in neuronal migration defects and early embryonic lethality. Nat Genet 1998;19:333339.

63 Liu Z, Steward R, Luo L: Drosophila Lis1 is required for neuroblast proliferation, dendritic elaboration and axonal transport. Nat Cell Biol 2000;2:776-783.

64 Feng Y, Olson EC, Stukenberg PT, et al: LIS1 regulates CNS lamination by interacting with mNudE, a central component of the centrosome. Neuron 2000;28:665-679.
65 Faulkner NE, Dujardin DL, Tai CY, et al: A role for the lissencephaly gene LIS1 in mitosis and cytoplasmic dynein function. Nat Cell Biol 2000;2:784-791.

66 Yan X, Li F, Liang Y, et al: Human Nudel and NudE as regulators of cytoplasmic dynein in poleward protein transport along the mitotic spindle. Mol Cell Biol 2003;23:1239-1250.

67 Selkoe D, Kopan R: Notch and presenilin: Reg ulated intramembrane proteolysis links development and degeneration. Annu Rev Neurosci 2003;26:565-597.

68 Lau KF, Howlett DR, Kesavapany S, et al: Cyclin-dependent kinase-5/p35 phosphorylates presenilin 1 to regulate carboxy-terminal fragment stability. Mol Cell Neurosci 2002;20:1320

69 Tezapsidis N, Merz PA, Merz G, et al: Microtubular interactions of presenilin direct kinesis of Abeta peptide and its precursors. FASEB J 2003:17:1322-1324.

70 Coquelle FM, Caspi M, Cordelières FP, et al: LIS1, CLIP-170's key to the dynein/dynactin pathway. Mol Cell Biol 2002;22:3089-3102.

71 Tai CY, Dujardin DL, Faulkner NE, et al: Role of dynein, dynactin, and CLIP-170 interactions in LIS1 kinetochore function. J Cell Biol 2002; 156:959-968.

72 Zhang Z, Hartmann H, Do VM, et al: Destabilization of beta-catenin by mutations in presenilin-1 potentiates neuronal apoptosis. Nature 1998;395:698-702.

73 Kesavapany S, Lau K, McLoughlin D, et al: p35/Cdk5 binds and phosphorylates beta-catenin and regulates beta-catenin/presenilin-1 interaction. Eur J Neurosci 2001;13:241-247.

74 Kwon YT, Gupta A, Zhou Y, et al: Regulation of N-cadherin-mediated adhesion by the p35Cdk5 kinase. Curr Biol 2000;10:363-372.

75 Schuman EM, Murase S: Cadherins and synaptic plasticity: Activity-dependent cyclin-dependent kinase 5 regulation of synaptic beta-catenin-cadherin interactions. Philos Trans R Soc Lond B Biol Sci 2003;358:749-756.

76 Wynshaw-Boris A, Gambello MJ: LIS1 and dynein motor function in neuronal migration and development. Genes Dev 2001;15:639_ 651.

77 Vallee RB, Tai C, Faulkner NE: LIS1: Cellular function of a disease-causing gene. Trends Cell Biol 2001;11:155-160.

78 Nikolic M, Dudek H, Kwon YT, et al: The $\mathrm{Cdk} 5 / \mathrm{p} 35$ kinase is essential for neurite outgrowth during neuronal differentiation. Genes Dev 1996;10:816-825.

79 Humbert S, Dhavan R, Tsai L: p39 activates Cdk5 in neurons, and is associated with the actin cytoskeleton. J Cell Sci 2000;113:975983.

80 dos Remedios CG, Chhabra D, Kekic M, et al: Actin binding proteins: Regulation of cytoskeletal microfilaments. Physiol Rev 2003;83:433473.

81 Ventura A, Pelicci PG: Semaphorins: Green light for redox signaling? Sci STKE 2002;2002: PE44.

82 Nikolic M: The role of Rho GTPases and associated kinases in regulating neurite outgrowth. Int J Biochem Cell Biol 2002;34:731-745. 
83 Papakonstanti EA, Stournaras C: Association of PI-3 kinase with PAK1 leads to actin phosphorylation and cytoskeletal reorganization. Mol Biol Cell 2002;13:2946-2962.

84 Sanders LC, Matsumura F, Bokoch GM, et al: Inhibition of myosin light chain kinase by p21-activated kinase. Science 1999;283:20832085.

85 Edwards DC, Sanders LC, Bokoch GM, et al: Activation of LIM-kinase by Pak1 couples Rac/ Cdc42 GTPase signalling to actin cytoskeletal dynamics. Nat Cell Biol 1999;1:253-259.

86 Wehrle-Haller B, Imhof BA: Actin, microtubules and focal adhesion dynamics during cell migration. Int J Biochem Cell Biol 2003;35:39_ 50.

87 Waterman-Storer CM, Worthylake RA, Liu $\mathrm{BP}$, et al: Microtubule growth activates Rac1 to promote lamellipodial protrusion in fibroblasts. Nat Cell Biol 1999;1:45-50.

88 Daub H, Gevaert K, Vandekerckhove J, et al: $\mathrm{Rac} / \mathrm{Cdc} 42$ and p65PAK regulate the microtubule-destabilizing protein stathmin through phosphorylation at serine 16 . J Biol Chem 2001;276:1677-1680.

89 Rashid T, Banerjee M, Nikolic M: Phosphorylation of Pak1 by the p35/Cdk5 kinase affects neuronal morphology. J Biol Chem 2001;276: 49043-49052.

90 Banerjee M, Worth D, Prowse DM, et al: Pak1 phosphorylation on $\mathrm{t} 212$ affects microtubules in cells undergoing mitosis. Curr Biol 2002;12: 1233-1239.

91 Lisman JE, Zhabotinsky AM: A model of synaptic memory: A CaMKII/PP1 switch that potentiates transmission by organizing an AMPA receptor anchoring assembly. Neuron 2001;31: 191-201.

92 Cukovic D, Lu GW, Wible B, et al: A discrete amino terminal domain of Kv1.5 and Kv1.4 potassium channels interacts with the spectrin repeats of alpha-actinin-2. FEBS Lett 2001; 498:87-92.

93 Wyszynski M, Lin J, Rao A, et al: Competitive binding of alpha-actinin and calmodulin to the NMDA receptor. Nature 1997;385:439-442.

94 Knudsen KA, Soler AP, Johnson KR, et al: Interaction of alpha-actinin with the cadherin/ catenin cell-cell adhesion complex via alphacatenin. J Cell Biol 1995;130:67-77.

95 Otey CA, Vasquez GB, Burridge K, et al: Mapping of the alpha-actinin binding site within the beta 1 integrin cytoplasmic domain. J Biol Chem 1993;268:21193-21197.

96 Pavalko FM, Otey CA, Simon KO, et al: Alphaactinin: A direct link between actin and integrins. Biochem Soc Trans 1991;19:1065-1069.

97 Blanchard A, Ohanian V, Critchley D: The structure and function of alpha-actinin. J Muscle Res Cell Motil 1989;10:280-289.

98 Walikonis RS, Oguni A, Khorosheva EM, et al: Densin-180 forms a ternary complex with the (alpha)-subunit of $\mathrm{Ca}^{2+} /$ calmodulin-dependent protein kinase II and (alpha)-actinin. J Neurosci 2001;21:423-433.

99 Rosenmund C, Westbrook GL: Calcium-induced actin depolymerization reduces NMDA channel activity. Neuron 1993;10:805-814.
100 Allison DW, Chervin AS, Gelfand VI, et al: Postsynaptic scaffolds of excitatory and inhibitory synapses in hippocampal neurons: Maintenance of core components independent of actin filaments and microtubules. $\mathbf{J}$ Neurosci 2000;20:4545-4554.

101 Lei S, Czerwinska E, Czerwinski W, et al: Regulation of NMDA receptor activity by Factin and myosin light chain kinase. $\mathbf{J}$ Neurosci 2001;21:8464-8472.

102 Woodring PJ, Hunter T, Wang JY: Inhibition of c-Abl tyrosine kinase activity by filamentous actin. J Biol Chem 2001;276:2710427110.

103 Woodring PJ, Litwack ED, O'Leary DD, et al: Modulation of the F-actin cytoskeleton by cAbl tyrosine kinase in cell spreading and neurite extension. J Cell Biol 2002;156:879-892.

104 Gertler FB, Doctor JS, Hoffmann FM: Genetic suppression of mutations in the Drosophila abl proto-oncogene homolog. Science 1990; 248:857-860.

105 Keshvara L, Magdaleno S, Benhayon D, et al: Cyclin-dependent kinase 5 phosphorylates disabled 1 independently of Reelin signaling. J Neurosci 2002;22:4869-4877.

106 Sheldon M, Rice DS, D'Arcangelo G, et al: Scrambler and yotari disrupt the disabled gene and produce a reeler-like phenotype in mice. Nature 1997;389:730-733.

107 Ware ML, Fox JW, Gonzalez JL, et al: Aberrant splicing of a mouse disabled homolog, mdab1, in the scrambler mouse. Neuron 1997;19:239-249.

108 Homayouni R, Rice DS, Sheldon M, et al: Disabled-1 binds to the cytoplasmic domain of amyloid precursor-like protein 1. J Neurosci 1999;19:7507-7515.

109 Ando K, Iijima KI, Elliott JI, et al: Phosphorylation-dependent regulation of the interaction of amyloid precursor protein with Fe65 affects the production of beta-amyloid. J Biol Chem 2001;276:40353-40361.

110 Sabo SL, Ikin AF, Buxbaum JD, et al: The Alzheimer amyloid precursor protein (APP) and FE65, an APP-binding protein, regulate cell movement. J Cell Biol 2001;153:14031414.

111 Ermekova KS, Zambrano N, Linn H, et al: The WW domain of neural protein FE65 interacts with proline-rich motifs in Mena, the mammalian homolog of Drosophila enabled. J Biol Chem 1997;272:32869-32877.

112 Krause M, Bear JE, Loureiro JJ, et al: The Ena/VASP enigma. J Cell Sci 2002;115: 4721-4726.

113 Zambrano N, Bruni P, Minopoli G, et al: The beta-amyloid precursor protein APP is tyrosine-phosphorylated in cells expressing a constitutively active form of the Abl protoncogene. J Biol Chem 2001;276:19787-19792.

114 Zhang B, Zelhof AC: Amphiphysins: Raising the BAR for synaptic vesicle recycling and membrane dynamics. Bin-AmphiphysinRvsp. Traffic 2002;3:452-460.
115 Messina S, Onofri F, Bongiorno-Borbone L, et al: Specific interactions of neuronal focal adhesion kinase isoforms with Src kinases and amphiphysin. J Neurochem 2003;84: 253-265.

116 Critchley DR: Focal adhesions - the cytoskeletal connection. Curr Opin Cell Biol 2000;12: 133-139.

117 Ferreira A, Rapoport M: The synapsins: Beyond the regulation of neurotransmitter release. Cell Mol Life Sci 2002;59:589-595.

118 Chi P, Greengard P, Ryan TA: Synapsin dispersion and reclustering during synaptic activity. Nat Neurosci 2001;4:1187-1193.

119 Chin LS, Li L, Ferreira A, et al: Impairment of axonal development and of synaptogenesis in hippocampal neurons of synapsin I-deficient mice. Proc Natl Acad Sci USA 1995;92: 9230-9234.

120 Ferreira A, Li L, Chin LS, et al: Postsynaptic element contributes to the delay in synaptogenesis in synapsin I-deficient neurons. Mol Cell Neurosci 1996;8:286-299.

121 Petrucci TC, Morrow JS: Actin and tubulin binding domains of synapsins Ia and Ib. Biochemistry 1991;30:413-422.

122 Benfenati F, Valtorta F, Chieregatti E, et al: Interaction of free and synaptic vesicle-bound synapsin I with F-actin. Neuron 1992;8:377386.

123 Fesce R, Benfenati F, Greengard P, et al: Effects of the neuronal phosphoprotein synapsin I on actin polymerization. II. Analytical interpretation of kinetic curves. J Biol Chem 1992;267:11289-11299.

124 Valtorta F, Greengard P, Fesce R, et al: Effects of the neuronal phosphoprotein synapsin I on actin polymerization. I. Evidence for a phosphorylation-dependent nucleating effect. J Biol Chem 1992;267:11281-11288.

125 Ceccaldi PE, Benfenati F, Chieregatti E, et al: Rapid binding of synapsin I to F- and Gactin: A study using fluorescence resonance energy transfer. FEBS Lett 1993;329:301305.

126 Valtorta F, Ceccaldi PE, Grohovaz F, et al: Fluorescence approaches to the study of the actin-nucleating and bundling activities of synapsin I. J Physiol Paris 1993;87:117-122.

127 Han HQ, Greengard P: Remodeling of cytoskeletal architecture of nonneuronal cells induced by synapsin. Proc Natl Acad Sci USA 1994;91:8557-8561.

128 Ceccaldi PE, Grohovaz F, Benfenati F, et al: Dephosphorylated synapsin I anchors synaptic vesicles to actin cytoskeleton: An analysis by videomicroscopy. J Cell Biol 1995;128: 905-912.

129 Baines AJ, Bennett V: Synapsin I is a microtubule-bundling protein. Nature 1986;319: 145-147.

130 Goldenring JR, Lasher RS, Vallano ML, et al: Association of synapsin I with neuronal cytoskeleton: Identification in cytoskeletal preparations in vitro and immunocytochemical localization in brain of synapsin I. J Biol Chem 1986;261:8495-8504. 
131 Bennett AF, Hayes NV, Baines AJ: Site specificity in the interactions of synapsin 1 with tubulin. Biochem J 1991;276(Pt 3):793-799.

132 Yang SD, Song JS, Hsieh YT, et al: Cyclic inhibition-potentiation of the crosslinking of synapsin I with brain microtubules by protein kinase FA (an activator of ATP.Mg-dependent protein phosphatase). Biochem Biophys Res Commun 1992;184:973-979.

133 Starr R, Xiao J, Monteiro MJ: Production of monoclonal antibodies against neurofilament-associated proteins: Demonstration of association with neurofilaments by a coimmunoprecipitation method. J Neurochem 1995;64:1860-1867.

134 Julien JP: Neurofilament functions in health and disease. Curr Opin Neurobiol 1999;9: 554-560.

135 Grant P, Sharma P, Pant HC: Cyclin dependent protein kinase $5(\mathrm{Cdk} 5)$ and the regulation of neurofilament metabolism. Eur J Biochem 2001;268:1534-1546.

136 Julien JP, Mushynski WE: Neurofilaments in health and disease. Prog Nucleic Acid Res Mol Biol 1998;61:1-23.

137 Collard JF, Cote F, Julien JP: Defective axonal transport in a transgenic mouse model of amyotrophic lateral sclerosis. Nature 1995; 375:61-64.
138 Williamson TL, Cleveland DW: Slowing of axonal transport is a very early event in the toxicity of ALS-linked SOD1 mutants to motor neurons. Nat Neurosci 1999;2:50-56.

139 Shea TB, Flanagan LA: Kinesin, dynein and neurofilament transport. Trends Neurosci 2001;24:644-648.

140 LaMonte BH, Wallace KE, Holloway BA, et al: Disruption of dynein(dynactin inhibits axonal transport in motor neurons causing lateonset progressive degeneration. Neuron 2002; 34:715-727.

141 Puls I, Jonnakuty C, LaMonte BH, et al: Mutant dynactin in motor neuron disease. Nat Genet 2003;33:455-456.

142 Hafezparast M, Klocke R, Ruhrberg C, et al: Mutations in dynein link motor neuron degeneration to defects in retrograde transport. Science 2003;300:808-812.

143 Zhao C, Takita J, Tanaka Y, et al: CharcotMarie-Tooth disease type 2A caused by mutation in a microtubule motor KIF1Beta. Cell 2001;105:587-597.

144 Ackerley S, Thornhill P, Grierson AJ, et al: Neurofilament heavy chain side arm phosphorylation regulates axonal transport of neurofilaments. J Cell Biol 2003;161:489-495.

145 Nguyen MD, Larivière RC, Julien JP: Deregulation of Cdk5 in a mouse model of ALS: Toxicity alleviated by perikaryal neurofilament inclusions. Neuron 2001;30:135-147.
146 Small JV, Kaverina I: Microtubules meet substrate adhesions to arrange cell polarity. Curr Opin Cell Biol 2003;15:40-47.

147 Manes T, Zheng DQ, Tognin S, et al: Alpha (v) beta-3 integrin expression up-regulates $\mathrm{cdc} 2$, which modulates cell migration. J Cell Biol 2003;161:817-826.

148 Guadagno TM, Ohtsubo M, Roberts JM, et al: A link between cyclin A expression and adhesion-dependent cell cycle progression. Science 1993;262:1572-1575.

149 Yamakita Y, Oosawa F, Yamashiro S, et al: Caldesmon inhibits Arp2/3-mediated actin nucleation. J Biol Chem 2003;278:1793717944.

150 Haas M, Jost E: Functional analysis of phosphorylation sites in human lamin A controlling lamin disassembly, nuclear transport and assembly. Eur J Cell Biol 1993;62:237-247.

151 Mary S, Charrasse S, Meriane M, Comunale F, Travo P, Blangy A, Gauthier-Rouviere C: Biogenesis of N-cadherin-dependent cell-cell contacts in living fibroblasts is a microtubuledependent kinesin-driven mechanism. Mol Biol Cell 2002;13:285-301.

152 Chausovsky A, Bershadsky AD, Borisy GG: Cadherin-mediated regulation of microtubule dynamics. Nat Cell Biol 2000;2:797-804. 